\title{
Geräuschangst bei Hunden und Katzen
}

\section{Entstehung, Prophylaxe und Therapie}

\author{
Celina del Amo
}

Geräuschängste bei Hunden und Katzen sind ein weit verbreitetes Problem, das zu bestimmten Jahreszeiten jeweils speziell Hochkonjunktur hat. Ein betroffenes Tier zeigt sich jedoch nicht nur in den „klassischen“ Problemsituationen wie Silvester, Karneval oder einem Schützenfest ängstlich, sondern durchlebt auch im Alltag wiederkehrend Phasen der Angst, wenn es mit den entsprechenden Problemreizen, d. h. den angstauslösenden Geräuschen, konfrontiert wird.

\section{Was ist Angst?}

Angst ist eine Emotion, die dem Körper als Schutzmechanismus dient bzw. dienen soll. Jeder Reiz, der über die verschiedenen Sinnesorgane (hier natürlich speziell die Ohren) wahrgenommen wird und einen individuellen Schwellenwert überschreitet, wird im limbischen System (einem Teil des Gehirns) in Sekundenbruchteilen mit einem emotionalen Attribut (einer Eigenschaft) versehen. Die Emotion Angst wird hierbei konkret im sogenannten Mandelkern ausgelöst, der zum limbischen System gehört. Im Normalfall lösen Reize Angst aus, die das Tier als Gefahr einordnet. Das bedeutet, diese Emotion hat eine wichtige Schutzfunktion. Im Fall von Geräuschängsten reagieren die Tiere jedoch mit Angst auf Reize, die auf das Leben des Tieres im Grunde keinerlei negativen Einfluss haben können, etwa auf einen Zischlaut oder auf einen Knall.

\section{Geräuschangst}

Die Palette an Problemgeräuschen kann von Tier zu Tier stark variieren. Bei einem Geräuschangstproblem ist daher vor dem Start einer Therapie von entscheidender Wichtigkeit, genau zu analysieren, welche Geräusche bei dem Patienten das Problemverhalten auslösen.

Geräuschängste können plötzlich auftreten, das heißt, das Tier muss zuvor keinerlei negative Erlebnisse mit diesem Geräusch gemacht haben. Typischerweise verschlimmern sich unbehandelte Geräuschängste über die Zeit und das Tier beginnt, den Problemreiz zu generalisieren. Das bedeutet, dass sich die Palette an Problemgeräuschen zunächst auf ähnliche Reize ausweitet. Nicht selten wird die Angst dann nach und nach auch auf andere Situationen übertragen, man spricht dann von einer Angstverknüpfung und Angstübertragung. Die Gefahr einer Angstverknüpfung ist umso größer, wenn das Tier bereits im Vorfeld in negativer Art mit dem angstauslösenden oder ähnlichen Geräuschen konfrontiert wurde, je weniger klar für das Tier zu erkennen ist, woher das Geräusch kommt oder wodurch es ausgelöst wird und je stärker seine generelle Stressbelastung im Moment des Geräuschereignisses ist.

Merke

Je später eine Therapie beginnt und je negativer ein angstauslösender Reiz empfunden wird, desto größer ist die Gefahr der Angstübertragung.

\section{Angstübertragung}

Ein Beispiel für eine Ausweitung der Angst wäre Folgendes: Der Hund erlebt zunächst panikartige Angst bei Schussgeräuschen. Anfänglich wird er vielleicht auf einem Spaziergang mit diesem Geräusch konfrontiert. Neben dem Geräusch selbst wird nicht selten auch der Ort mit der Angst assoziiert, sodass der Hund beispielsweise beginnt, konkrete Wegstrecken zu meiden. Als nächstes wird das Problem möglicherweise auf Feuerwerke übertragen, da auch hier Schusslaute eine Rolle spielen. Darüber hinaus kommen nun aber auch Zischlaute und laute dumpfe Töne hinzu. Die Angst vor Zischlauten kann dann auf die Situation mit einem Heißluftballon oder zuhause auf das Öffnen einer Flasche mit Kohlensäure überspringen und von dort weiter auf andere Haushaltsgeräte oder draußen auf Flugobjekte, wie etwa 
Flugzeuge oder Lenkdrachen und von hier wiederum auf Fahnen, die im Wind flattern und weiter auf Wind im Allgemeinen und so weiter und so fort. Die lauten dumpfen Töne aus diesem Beispiel wären ein Einstieg für die Übertragung der Angst auf Schützenfeste, Verkehrssituationen oder auch Angst vor Menschenmengen.

\section{Angst und Panik}

Geräuschangstprobleme können in unterschiedlichen Schweregraden auftreten. Schon beim Erkennen beginnenden Stresses in Geräuschsituationen, der sich anfänglich eher als Schüchtern- oder Unsicherheit zeigt, sollte das Problem therapeutisch angegangen werden, um eine Ausweitung zu vermeiden. Leidet das Tier bereits unter starker Angst/Furcht, sind weitere Stressanzeichen wie Unkonzentriertheit, Meideverhalten und vegetative Symptome wie etwa das Hecheln, eine gesteigerte Herzschlagrate, schwitzige Pfoten etc. zu beobachten. Der schlimmste Ausprägungsgrad des Problems ist die Panik, wobei das Tier zu fliehen versucht. Seltener, speziell bei Berührungen oder körperlichen Einschränkungen des Tieres, kann auch aggressives Verhalten ausgelöst werden.

\section{Unbedachter Strafeinsatz}

Leider wird immer wieder bei der Erziehung von Hunden und Katzen propagiert, geräuschvolle Strafen einzusetzen. Hierzu zählen Maßnahmen wie das Klappern oder Werfen mit Klapperdosen, Wurfketten, lautes In-die-Hände-Klatschen, geräuschvolles Zischen, Anschreien oder auch der Einsatz von Sprühhalsbändern (bei Hunden). In aller Regel ist das Ziel der Strafanwendung, dass das Tier ein bestimmtes Verhalten nicht mehr zeigen soll. Übersehen wird hierbei jedoch ein wesentliches Detail, das der Entstehung eines Geräuschangstproblems nicht selten Vorschub leistet: Die Maßnahme kann nur dann im Sinne einer Strafe „erfolgreich“ sein, wenn sich das Tier ängstigt, andernfalls wird es sein Handeln nicht unterbrechen. Konkret wird bei derartigen Strafen 
also zwangsläufig das Gefühl der Angst mit dem jeweiligen Geräusch verknüpft ein ,idealer“ Einstieg für die Entwicklung eines Geräuschproblems. Zum anderen fühlt sich das Tier häufig chancenlos, vor allem, wenn es nicht zuvor bereits eine andere und erfolgsverknüpfte Lösungsstrategie erlernt hat. Es weiß in diesem Fall nämlich nicht, was zur Strafe geführt hat und wie es der Strafe zukünftig entkommen kann.

Merke

Der Einsatz von Geräuschen zu Erziehungszwecken kann die Entwicklung einer Geräuschangst fördern, weil hierbei Geräusche mit Angst verknüpft werden.

\section{Prophylaxemaßnahmen}

Aufgrund der erst kurzen Lebensspanne können Jungtiere in aller Regel zunächst nur über einen vergleichsweise geringen Erfahrungsschatz mit Geräuschsituationen zurückblicken. Folgende Maßnahmen sind daher sinnvoll, um junge Tiere schrittweise auf verschiedene Geräuschsituationen vorzubereiten und Angstverhalten vorzubeugen:

- Jungtiere sollten im Falle eines vorhersehbaren Ereignisses wie etwa einem Feuerwerk nicht unbetreut gelassen werden. Ebenfalls gilt es, sie keinesfalls unbedacht mit Geräuschsituationen zu konfrontieren, da die Reaktionen des Tieres im Vorhinein nicht eingeschätzt werden können.

- Ein Jungtier sollte während einer Geräuschsituation möglichst nie mit einem geräuschängstlichen älteren Tier zusammen sein, um ein „Überspringen“ der Stimmung zu vermeiden, denn junge Tiere schauen sich besonders leicht Verhaltensweisen von Sozialpartnern ihrer eigenen Art ab, wenn sie mit diesen während einer Problemsituation in engem Kontakt stehen.
- Die Anwesenheit eines entspannten und angstfreien (älteren) Tieres kann hingegen durchaus gezielt als sinnvolle, prophylaktische Maßnahme zur Beruhigung eines Jungtieres eingesetzt und als positiver Einfluss genutzt werden.

\section{Positive Verknüpfung}

Um zu erreichen, dass die Tiere bei der Konfrontation mit lauten Geräuschen im Alltag entspannt bleiben können, ist es sinnvoll, Geräusche mit etwas Positivem zu koppeln und dabei möglichst nicht viel Aufhebens um die Sache zu machen. Eine der einfachsten Möglichkeiten ist es, die Tiere in der Folge eines Geräuschereignisses bzw. während dieses auftritt, in neutraler Art auf ein ihnen vertrautes Spiel mit Futter zu lenken. Aber auch andere Spiele oder Beschäftigungen, die den Tieren Freude bereiten und deren Konzentration verlangen, können zu diesem Zweck genutzt werden, da das Abgleiten in Angst während einer konzentrationsintensiven „Arbeit“ nicht möglich ist. Eine ideale Form des prophylaktischen Geräuschtrainings ist, das Tier dazu zu animieren, selbst laut zu spielen, denn selbsterzeugte Geräusche lösen aufgrund der klar erkennbaren Herkunft in aller Regel keine Ängste aus. Mit Trockenfutter befüllbare Spielzeuge aus Plastik oder PET-Flaschen erfüllen diesen Zweck in idealer Art.

\section{Strafen vermeiden}

Ein häufig benanntes Erziehungsziel ist das „Unterlassen“ einer Handlung. Jedoch ist dies ein für Hunde und Katzen generell zu abstraktes Bild für einen schnellen Lernerfolg. Grundsätzlich gilt: Tiere erst eine unerwünschte Handlung ausführen zu lassen, um diese dann zu bestrafen, ist kein kluger Schachzug! Schließlich wurde die Handlung ja dennoch „geübt“. Wesentlich sinnvoller ist es hingegen, ihnen über einen kleinschrittigen und erfolgsbasierten Trainingsansatz eine für die jeweilige Situation passende (alternative) erwünschte Verhaltensweise beizubringen, denn in diesem Fall wird das Tier das erwünschte Verhalten auch zukünftig gerne umsetzen. Wer diesen Weg geht, wird praktisch niemals in die Verlegenheit kommen, geräusch-verknüpfte (oder andere grundsätzlich immer Angst oder Schmerz erzeugende) Strafen anzuwenden.

\section{Fallanalyse}

Hunde und Katzen tendieren stärker zu Ängsten und möglichen neuen Angstverknüpfungen, je höher ihre individuelle Stresslast ist. Bei der Abklärung eines Geräuschangstpatienten ist die Erstellung einer detaillierten Liste der Problemgeräusche nur der erste Schritt. Ebenfalls wichtig ist die Analyse auf medizinischer und sozialer Ebene. Hierbei muss oftmals regelrecht detektivische Arbeit geleistet werden, um sämtliche negativen Stressoren, die auf das Tier einwirken, aufdecken und in der Therapie berücksichtigen zu können. Nur ein umfassender klinischer Check-up (inklusive der Überprüfung auf das Vorliegen von Schmerzen, einer Entzündungsquelle oder Erregerbelastung, Erkrankungen von Leber, Nieren, Herz und Schilddrüse) kann einen stichhaltigen Aufschluss bringen. Gleiches gilt für die umfassende Kontrolle im Hinblick auf die Lebenssituation und das Training mit dem Tier.

\section{Therapiemaßnahmen}

Im Umgang mit einem unter Ängsten leidenden Tier hilft es generell, Ruhe und souveräne Freundlichkeit auszustrahlen, um dem Tier so auch in potenziell schwierigen Situationen ein Fels in der Brandung zu sein. Körperliche Nähe wird von ängstlichen Tieren mitunter eher als zusätzliche Bedrohung statt als Unterstützung wahrgenommen. Ein schneller Test kann hier Aufschluss bringen: Wenn sich das Tier bei der körperlichen Berührung (s. Tipp) nicht merkbar entspannt (bemerkbar an der Normalisierung von Herzschlagrate und Atemfrequenz), ist von dieser Art der Zuwendung abzusehen! Ähnliches gilt für verbale Beruhigungsversuche. 
Tipp für die Durchführung des Tests: Die meisten Patienten reagieren auf einen ruhigen und konstanten Körperkontakt wie das Auflegen einer Hand an einer dem Tier angenehmen Stelle eher mit Entspannung als beim aktivierenden Kraulen oder Streicheln!

\section{Gegenkonditionierung}

Für eine langfristige Therapie stellt die sogenannte klassische Gegenkonditionierung eine der wichtigsten Therapieübungen dar. Hierbei werden die Angstemotionen durch eine fortgesetzte Kopplung des Problemreizes an einen extrem hochwertigen und positiv belegten Reiz (z. B. Lieblingsfutter) nach und nach in Neutralität und später ggf. auch in Freude „ausgetauscht“. Um überhaupt eine positive Emotion kreieren zu können, ist es häufig vor allem zu Beginn der Therapie entscheidend, die Intensität des Pro-

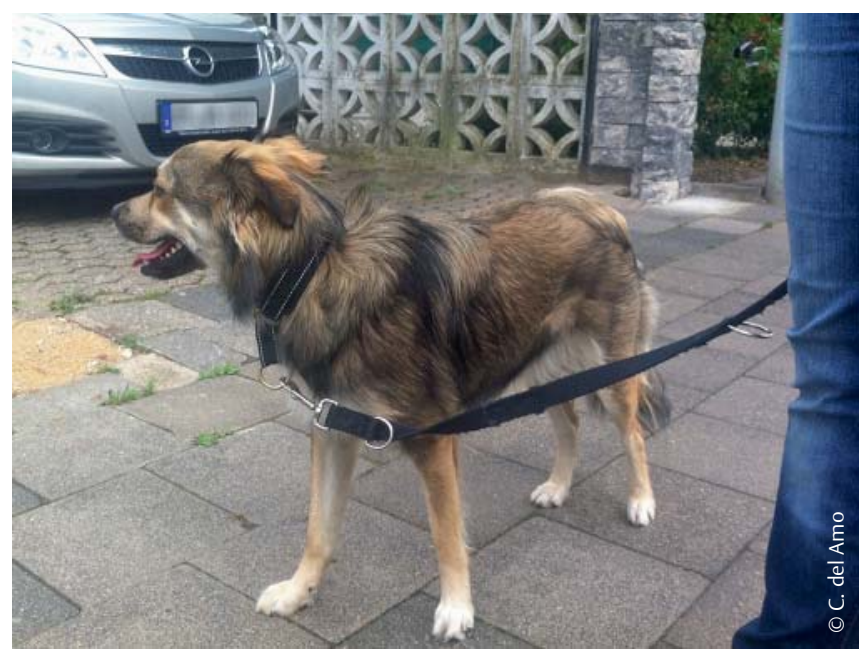

Abb. 1 Bei vorhersehbaren Geräusch-Ereignissen gilt zu deren Schutz Leinenpflicht für Junghunde und geräuschängstliche Tiere.

blemgeräusches zu minimieren und das zur Kopplung eingesetzte Futter wirklich aus der Kategorie der Lieblingssnacks zu entnehmen. Langfristig zählt für den Therapieerfolg der Fleiß bei der Umsetzung der Übungen, denn um eine Angstemotion in Freude verwandeln zu können, sind mehrere Tausend Wiederholungen erforderlich!

\section{Panik verhindern}

Ein sehr wichtiger Punkt im Umgang mit Geräuschangstpatienten ist die relativ große Gefahr, in Belastungssituationen in Angst und Schrecken wegzulaufen. Auch bei sonst gutem Gehorsam sind Hunde, die in Panik geraten, dann beispielsweise nicht mehr in der Lage, zuverlässig auf Kommandos zu reagieren. In Situatio- 


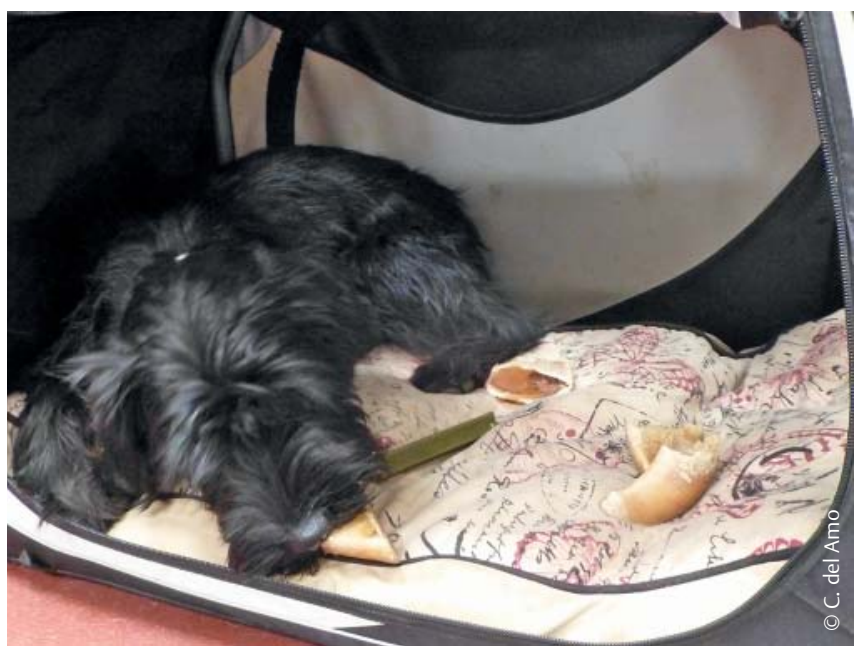

Abb. 2 Ein fester, positiv verknüpfter Rückzugsort (z. B. Hundebox) hilft ängstlichen Tieren bei der Bewältigung von Stresssituationen.

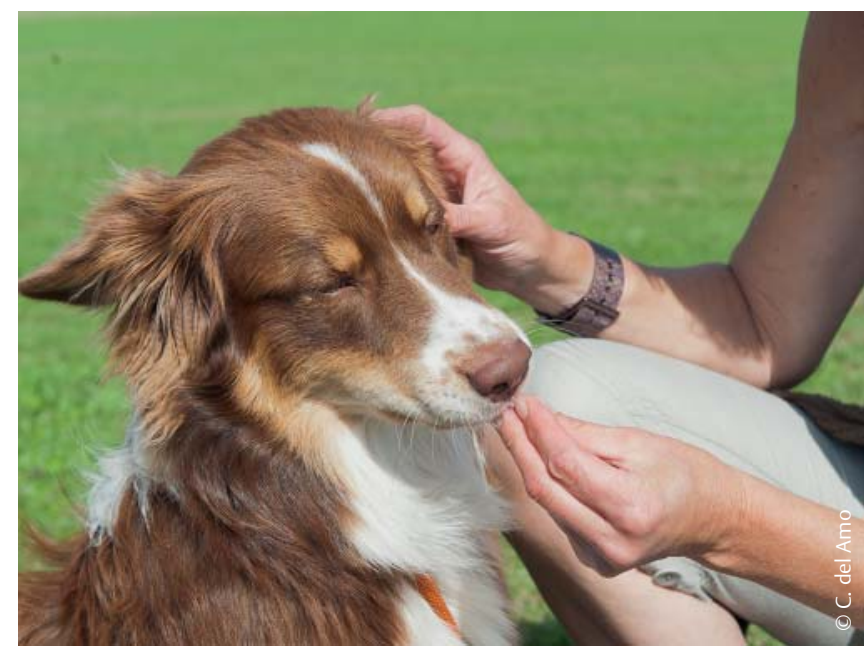

Abb. 3 Berührungen an den Ohren müssen dem Hund über positive Verknüpfungsübungen vertraut gemacht werden, bevor schallschützende Hilfsmittel eingesetzt werden können. nen, in denen es in vorhersehbarer Art zu einem belastenden Geräuschereignis kommen wird (etwa bei einem zuvor angekündigten Feuerwerk o. Ä.), gilt daher für Hunde draußen zur Sicherheit durchgehende Leinenpflicht ( $\bullet$ Abb. 1), die vorübergehende Reduktion der Spaziergänge auf die Versäuberung und für Freigängerkatzen die strikte Beschränkung des Freigangs.

\section{Umgebung anpassen}

Alternative Indoor-Aktivitäten (Spiele, Denksportaufgaben, Körperpflege - sofern das Tier dies genießt) sind in diesen Momenten sinnvolle Maßnahmen zum Ausgleich für die Reduzierung des Auslaufs im Freien. Sie helfen zudem den Tieren, den Fokus vom Problemreiz weg auf eine Spaßaktivität zu lenken, bzw. sie grundsätzlich vom Problemreiz abzulenken.

Geräuschängstliche Tiere sollten zuhause freien Zugang zu einem speziellen Rückzugsort haben ( $\bullet$ Abb. 2). Wenn zu erkennen ist, dass sich das Tier gerne verstecken möchte, sind abgeschottete Plätze ideal. Wichtig ist hier, dass diese Orte dem Tier in problemfreien Momenten bereits als
„Trainingsinhalt“ positiv vertraut gemacht wurden, denn das Ziel ist eine Verknüpfung mit dem Gefühl von Geborgenheit und Entspannung.

Auch die Einschränkung der Geräuschbelastung mittels fest verschlossener Türen und Fenster (ggf. mit herabgelassenen Jalousien) während einer von außen einwirkenden Problemsituation (Feuerwerk, Wind) stellt eine Hilfe für die Tiere dar. Bei Hunden kann die Geräuschbelastung, nach einem entsprechenden Training, auch durch die Anwendung von SilikonOhrstöpseln, speziellen Kopfhörern oder Bandagen gemindert werden ( $\bullet \mathbf{A b b} \mathbf{3} \mathbf{3})$.

\section{Medikation}

Bei schweren Ängsten stellt der kurzfristige Einsatz eines angstlösenden Beruhigungsmittels (aus der Gruppe der Benzodiazepine) einen wichtigen Therapiebaustein dar, um zu verhindern, dass sich das Problem noch weiter ausweitet. Dieser Ansatz macht sich speziell in den Fällen bezahlt, in denen es sich um ein akutes und noch unbehandeltes Problem handelt oder eine begonnene Therapie noch nicht weit genug fortgeschritten ist.
Auch die längerfristige therapiebegleitende Verschreibung eines Psychopharmakons durch einen erfahrenen tiermedizinischen Verhaltenstherapeuten ist in manchen Fällen angezeigt und im Bedarfsfall in akuten Momenten durch ein als Add-on eingesetztes Benzodiazepin zu ergänzen.

\section{Weitere Präparate}

Auch „Wohlfühl“-Pheromone wie Feliway $^{\circledR}$ oder ADAPTIL ${ }^{\circledR}$ können therapiebegleitend Anwendung finden. Als alleinige Maßnahme sind diese Mittel jedoch nur in Fällen milder Belastungen, die sich eher in Unsicherheit und keinesfalls als Angst oder gar Panik äußern, ausreichend. Ähnliches gilt auch für den Einsatz bestimmter Futtermittel (z. B. Calm ${ }^{\circledast}$ ) bzw. Futtermittelzusätze (z. B. Zylkène ${ }^{\circledR}$, Calmex ${ }^{\circledR}$ oder Adaptil Tabletten $^{\circledR}$ ).

Online

http://dx.doi.org/10.1055/s-0035-1568270

\section{Celina del Amo}

Lupologic $\mathrm{GmbH}$

Linienstr. 72

40227 Düsseldorf 